\title{
Viewpoint
}

\section{Spectrum of care: more than treatment options}

\author{
Carolyn R. Brown DVM \\ Laura D. Garrett DVM \\ William K. Gilles DVM \\ Kendall E. Houlihan DVM \\ Emily McCobb DVM, MS \\ Sharon Pailler PhD, MPA \\ Holly Putnam DVM \\ Jennifer L. Scarlett DVM \\ Linda Treglia DVM \\ Brittany Watson VMD, PhD \\ Henry T. Wietsma DVM, MS
}

\begin{abstract}
From the American Society for the Prevention of Cruelty to Animals, New York, NY 10018 (Brown, Pailler); Department of Veterinary Clinical Medicine, College of Veterinary Medicine, University of Illinois, Urbana, IL 61802 (Garrett); Access Veterinary Care, Minneapolis, MN 55422 (Gilles); Animal Welfare Division, AVMA, Schaumburg, IL 60173 (Houlihan); Department of Clinical Sciences and Center for Animals and Public Policy, Cummings School of Veterinary Medicine, Tufts University, North Grafton, MA 01536 (McCobb); Emancipet, Austin, TX 78752 (Putnam); San Francisco Society for the Prevention of Cruelty to Animals, San Francisco, CA 94103 (Scarlett); Island Rescue, Bayshore, NY II706 (Treglia); School of Veterinary Medicine, University of Pennsylvania, Philadelphia, PA 19104 (Watson); and Pets in Need Veterinary Clinic, Riverside, RI 02915 (Wietsma).
\end{abstract}

Address correspondence to Dr. Brown (carolyn.brown@aspca.org).
$\mathbf{T}$ he past several decades have seen substantial advances in clinical veterinary medicine, including widespread specialization, access to more complex diagnostic testing and imaging methods, and increased availability of advanced treatments. However, these advances, in conjunction with the increasing costs of veterinary education, medical equipment, and general practice operations, mean that veterinary care can be financially out of reach for many pet owners. ${ }^{1} \mathrm{~A}$ recent survey ${ }^{2}$ of pet owners in the United States found that $28 \%$ had experienced a barrier to veterinary care in the previous 2 years and that the overwhelming barrier, for all types of care, was financial. According to the Humane Society of the United States, ${ }^{3} 88 \%$ of pets currently living in underserved communities in the United States have not been spayed or castrated, and financial considerations presumably play a large role in this low neutering rate.

According to the report of a 2018 Access to Veterinary Care Coalition survey, ${ }^{2} 95 \%$ of US veterinarians surveyed agreed or strongly agreed with the statement that "all pets deserve some level of veterinary care," and $98 \%$ of veterinarians employed in private practice reported they had implemented at least one strategy in the past year to address and mitigate financial barriers to veterinary care. According to the report, ${ }^{2}$ " $[\mathrm{t}] \mathrm{he}$ most frequently cited strategy was exploring treatment options with clients to best match their economic constraints." This suggests that most veterinarians in small animal clinical practice, whether they know it or not, are already providing what has come to be called an SoC.

As indicated by Stull et al, ${ }^{4}$ "[v] eterinarians have a wide spectrum of diagnostic and treatment options

\section{ABBREVIATIONS}

SoC Spectrum of care they can provide for their patients," ranging "from technologically advanced and expensive interventions to less advanced and less costly options." "Providing a continuum of acceptable care that considers available evidence-based medicine while remaining responsive to client expectations and financial limitations" ${ }^{5}$ is the cornerstone of SoC. However, for SoC to be truly effective at increasing access to veterinary care, both veterinarians and clients must view the care options being offered as viable and potentially desirable choices. Importantly, veterinarians must feel confident in discussing the advantages and disadvantages of each of the various options offered. Also, in human medicine, the doctor's recommendation can be the biggest influence when people are deciding among treatment options for themselves, ${ }^{6}$ and it seems likely that veterinarians have a similar influence on pet owners. Therefore, how care options are presented can be as important as which care options are presented.

In providing an $\mathrm{SoC}$, veterinarians have a responsibility to provide context for each option, discussing the advantages, disadvantages, and most likely outcomes for each option; the possibilities of favorable and unfavorable outcomes; the likelihood that additional testing or treatment might be needed; the associated costs; and the strength of the supporting evidence. ${ }^{7}$ Veterinarians should empower owners to choose the care option that best fits their expectations and financial considerations, without making them feel they are failing their pet if the most intensive, most expensive, or most technologically advanced option is not chosen. For many conditions, there is not a single treatment option that will routinely result in the best outcome or there is insufficient research or evidence to determine which treatment option will result in the best outcome. ${ }^{8}$ Additionally, many 
factors play a role in determining which outcome (eg, survival time vs quality of life) is the "best" outcome and, thus, which care option is truly the best option for any specific patient and its owners.

Offering and providing an SoC can be difficult but can also provide substantial benefits for all stakeholders in the veterinarian-client-patient relationship. In this report, we discuss barriers veterinarians face in effectively providing an SoC, including professional perceptions, attitudes, and misunderstandings related to the evidence supporting various care options, and present specific strategies for practicing an SoC. We also discuss the essential need for developing strong veterinarian-client relationships and fostering core communication skills, and we highlight how good communication skills contribute to the effective practice of an SoC. We encourage veterinarians, veterinary teams, and the veterinary profession as a whole to reexamine how expanding diagnostic testing, treatment, and management options can improve individual patient and community welfare.

\section{Veterinarians: Challenges to Providing an SoC}

In our experience, many veterinarians practice with the goal of providing the most intensive and most technologically advanced treatments. This may, at least in part, reflect their extensive exposure to specialists during their veterinary school training, in that clinical rotations for veterinary students in the United States have historically taken place at tertiary referral teaching hospitals. However, patients seen at traditional veterinary teaching hospitals often have uncommon or complex medical conditions, and owners typically arrive with the expectation that their pets will receive state-of-the-art treatments with a concomitant high cost. ${ }^{9}$ Although many veterinary teaching hospitals also run busy emergency services, cases are often referred to specialty services following admission. Thus, there frequently is an assumption that owners will want to pursue expansive diagnostic testing and advanced treatment for their pets. As a result, many veterinarians were not exposed to the SoC concept during their veterinary school clinical rotations, making it likely that they never had an opportunity to learn or gain confidence in the skills necessary to offer an SoC. ${ }^{10}$ Veterinary schools have begun to expand their approach by also providing training in community practices, allowing students to experience different types of practice, including practices offering an SoC. ${ }^{11}$ Still, the lack of training in or familiarity with the SoC concept means that veterinarians may be anxious about offering any treatment options other than the most intensive and most technologically advanced, worrying that doing so could adversely affect their reputation among peers and clients, result in litigation, or brand them as a substandard practitioner.

There are, however, many strategies for incorporating an SoC into treatment planning. For example, if a diagnostic test will provide information about a pet's disease, but not change the treatment plan or prognosis, the veterinarian may elect to forego that particular diagnostic test, depending on the owner's desire to have a definitive diagnosis. In addition, testing and treatments could be applied in a sequential approach, with limited testing and empirical or conservative treatments used first and additional testing and more complex treatments used depending on the pet's response.

Ongoing research supports the established principle that a variety of treatment approaches can represent good medical care and lead to successful outcomes. ${ }^{12}$ One area of SoC research considers whether patients can successfully be treated as outpatients for conditions that have traditionally been thought to require hospitalization. An example of this is the Colorado canine parvovirus protocol, which has been reported as effective in clinical practice. ${ }^{13,14}$ Another area of SoC research explores whether implementing more aggressive and more costly treatments early in the course of disease decreases overall pain for the pet and cost for the owner. An example of this approach might be performing a perineal urethrostomy after the first or second episode of urinary obstruction rather than waiting for a third episode. ${ }^{15}$ For other conditions such as pyometra, discussing total cost and risks associated with having the surgery performed at a general practice versus a specialty or emergency hospital can assist owners in making decisions about which option to choose. ${ }^{16,17}$

Veterinarians may face scrutiny by a segment of their peers when offering care options from the less intensive and expensive end of the $\mathrm{SoC}^{18}$ or may worry that they will be perceived as cutting corners, resulting in doubt and shame about the care they provide. This attitude can persist even when treatment is successful and when the alternative would have been that the pet did not receive any care at all. Judging colleagues harshly creates tension within the profession and fosters a reluctance to share experiences that might benefit patients. Veterinarians can support each other by creating networks of likeminded peers who discuss increasing access to veterinary care and providing an SoC for their clients and patients. ${ }^{19}$ Sharing experiences and learnings in a supportive environment can reinforce the value of offering an SoC.

It is possible that some veterinarians default to offering the most intensive and most technologically advanced treatments because of liability concerns or concerns about losing their license to practice if they were to offer other options that they perceive as not meeting the so-called standard of care. Most state veterinary practice acts require that veterinarians practice in compliance with the standard of care. However, "standard of care" is a legal term defined as "care required of and practiced by the average reasonably prudent, competent veterinarian in the community." 20 This definition is vague and can vary over 
time and across geographic locations ${ }^{21}$; however, it does not require that general practitioners provide the same level of care as specialists or dictate that the most intensive and most technologically advanced care is the only acceptable care. Complaints filed with state veterinary boards can result in fines or suspension or revocation of a veterinary license. However, good veterinarian-client communication can help avoid most of the misunderstandings that lead to veterinary board complaints, and good medical record keeping that documents discussions with owners and clarifies that owners were fully informed before deciding on a care option can be the best defense against them.

Today's social media climate can also sometimes be seen as a barrier to practice an SoC, because social media allows the voice of a single unhappy client claiming their pet did not receive appropriate care to be greatly magnified. Approximately one in five veterinarians report having been victims of cyberbullying or knowing colleagues who were victims of cyberbullying. ${ }^{22}$ On the other hand, social media has the potential to promote a more-balanced discussion of the costs of veterinary care and options available under an SoC.

\section{Clients: Communication Strategies}

Communication that focuses on building the veterinarian-client relationship is an essential component of SoC. Specific relationship-centered communication skills include asking open-ended questions and employing reflective listening, which have been shown to improve the client experience and increase their satisfaction with veterinary services by creating a positive base for the transfer of information from veterinarian to client and enhancing information retention and compliance with treatment protocols. ${ }^{23-25}$ These core communication skills are being incorporated into many veterinary school curricula ${ }^{26,27}$ and continuing education opportunities for practicing veterinarians. Relationship-centered communication decreases the likelihood of a client taking legal or disciplinary action against a practitioner or posting a negative review by preventing misunderstandings or misaligned expectations, ${ }^{28}$ which may alleviate some barriers to practicing SoC.

Listening carefully to clients and allowing them to relay their observations of their pet and voice their concerns is an important step in building trust ${ }^{29}$ and developing appropriate SoC options. Practicing reflective listening, which includes summarizing what another person has said and checking that you have understood them correctly, ${ }^{30,31}$ reduces the likelihood of miscommunication and may save time and frustration during the course of a pet's treatment. ${ }^{32}$

Asking open-ended questions (ie, questions that require more than a yes-or-no, single word, or short answer) encourages clients to share more about their pet's condition and their desires and limitations related to their pet's care. ${ }^{30,31}$ The answers to these types of questions provide important context when discussing various SoC options. Questions that are not open-ended may make owners feel judged or pressured to answer with what they believe is the "correct" answer even if it is not the best answer for their situation. For example, clients may answer "yes" when asked whether they can give their dogs injections, even if they have reservations about their ability to do so. If an open-ended question is asked ("How would you feel about giving injections at home?"), clients will be more likely to provide information critical to determining the treatment plan.

Discussing the family's goals when caring for their pet allows veterinarians to better determine what will be considered a successful outcome from the family's perspective and which care options are most likely to achieve that outcome. During a goalsof-care discussion, veterinarians should ask questions such as "what are your biggest concerns about your pet's condition?," "how do you think your pet will tolerate being in the hospital or returning for multiple visits?," "what is your ability to come back for recheck examinations?," and "what is your budget for your pet's care?" A helpful tool to assist with navigating these conversations is the conversation guide from Goldberg, ${ }^{33}$ which provides a structure for difficult conversations. Allowing clients time to process information and prepare for the next phase of a discussion is important. Checking in with owners, asking whether they are ready to continue a difficult conversation, and using questions to ensure understanding indicates that veterinarians are sensitive to owners' feelings and willing to spend the time necessary to make them feel at ease. ${ }^{34}$

Including owners in the process of winnowing down the universe of potential care options to those most closely aligned with their goals, expectations, abilities, and financial considerations leads to better decisions for pets by empowering the family to consider what is possible and in their pets' best interests. Family considerations include the initial and longterm costs, logistics of ongoing treatment, and medical factors such as diagnosis and prognosis. ${ }^{35}$ Families may consider their pets' individual characteristics, including temperament, which can affect an owner's ability to comply with treatment recommendations and the pets' quality of life (eg, because of extended or multiple hospitalizations, intensive treatments, or activity restriction). In addition, owners may consider their own lifestyle factors such as the ability to physically perform treatments, return for recheck appointments, or provide special support at home for their pets (Figure I)

Even without any outside bias or intentional inference, most people will, when faced with a list of options, tend to gravitate toward the first option offered. ${ }^{36}$ Thus, if veterinarians present the most intensive and most technologically advanced care option 


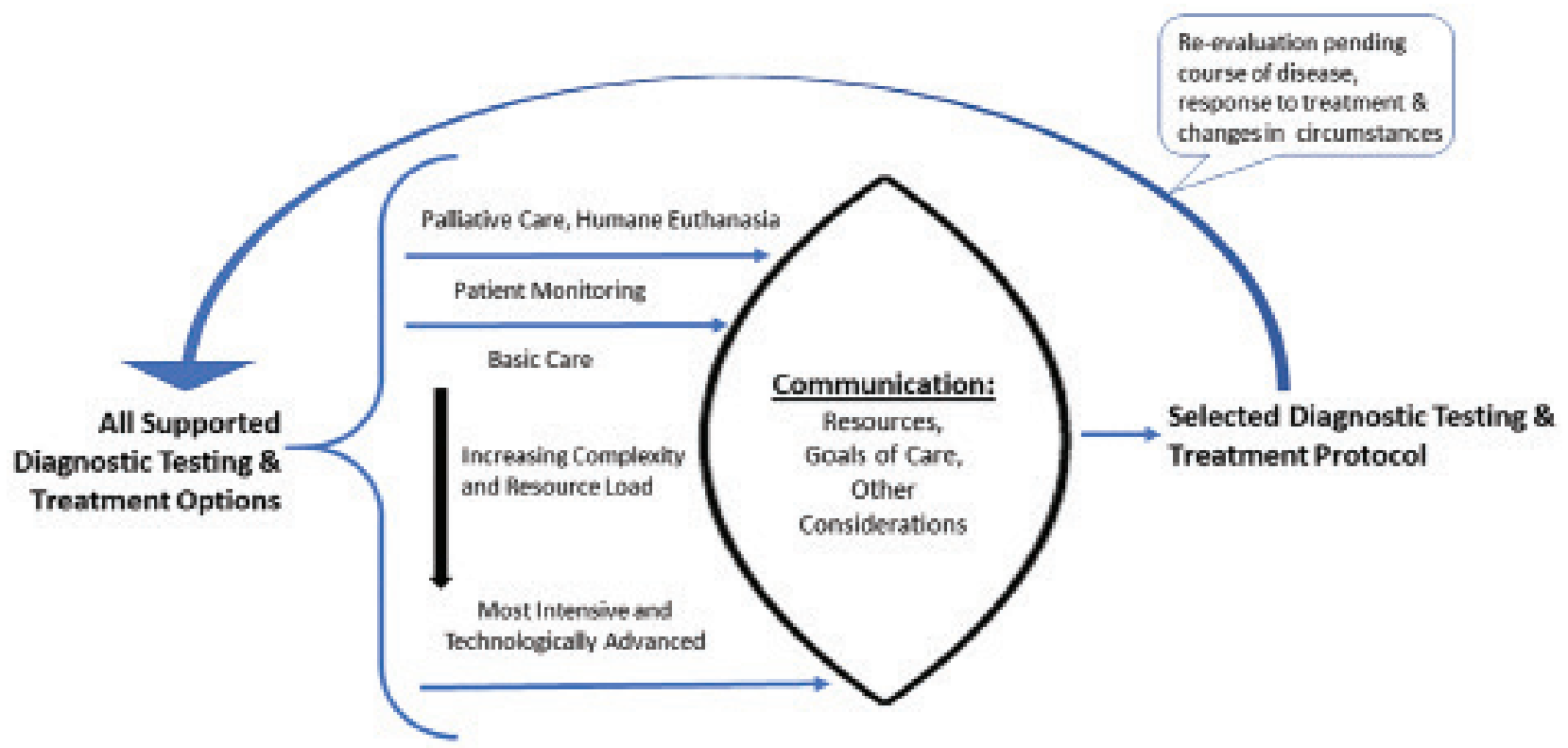

Figure I-Illustration of the spectrum of care in veterinary medicine. All evidence-supported diagnostic testing and treatment options are filtered through the lens of the specific circumstances of the pet and owner to select the option considered most acceptable on the basis of the owner's expectations and financial considerations. Care options should be periodically reviewed to ensure that they continue to meet the owner's expectations.

first, owners are likely to infer that this is the preferred or possibly only acceptable option. With the benefit of insights gained through a goals-of-care discussion, veterinarians can instead first offer the option they believe will be the best for the pet on the basis of current scientific knowledge ${ }^{37}$ and the owners' expectations and financial considerations.

It is of paramount importance for clients to be able to communicate with their veterinarian in a language in which they are comfortable. Conversations about a pet's medical condition and care are often complex and emotional, may include information that is new to the client, and could contain unfamiliar concepts and terminology. Navigating that conversation in a language in which the client is not fluent adds to the stress of the situation and makes good decision-making more difficult. If possible, having staff who can translate care conversations, including medical terms, into languages that are common in the practice area is optimal; however, other methods of translation, such as various websites and contracted telephone translation services, are available. Infographics that depict important information may also be helpful.

\section{Patients: Improving Welfare}

Patients are the ultimate beneficiaries of offering an SoC, and offering an SoC is the first step in improving pet welfare for clients having difficulties accessing care. Clients, especially those who have never or rarely brought their pets to see a veterinarian, must feel comfortable seeking veterinary care and at ease during their visit to ensure an ongoing relationship with the clinic. Participating in community events, hosting an open house at the clinic, or speaking at community meetings are ways veterinarians can help potential clients become more familiar with them and their practices and decrease the apprehension some clients may feel about bringing their pets to a clinic. When possible, employing veterinarians and staff who are members of the community and who have similar cultural, racial, and socio-economic experiences will not only help make clients feel more comfortable in the clinic but also build sensitivity and a welcoming atmosphere for clients.

Anxiety over the cost of veterinary care may make some clients reluctant to bring their pets to a veterinary clinic. ${ }^{32}$ Demystifying fees can help to allay this anxiety. ${ }^{38}$ Transparency about the cost of basic services such as the initial examination fee prior to the visit can help set expectations and help clients prepare. Openly discussing the costs of various treatment plans early in the process is an essential component of providing an SoC, yet one study ${ }^{39}$ suggested that $<30 \%$ of veterinarians discussed cost with their clients during their visits. Veterinarians should be able to articulate how the costs of various diagnostic tests and treatments compare, explain why some are more expensive than others, and discuss the expected benefit or outcome for each. ${ }^{7}$ Price transparency is key for building trust with clients. ${ }^{38}$ One strategy that can be used to simplify estimating fees is to offer inclusive packages with fixed pricing for protocols and procedures that generally require the same approach for every patient. This approach makes it easier for clients to compare costs of different treatments.

Decreasing the cost of care by practicing an SoC has many benefits for patients. Pets will be treated for conditions that might otherwise have not been 
addressed. Clients may pursue treatment for a future condition earlier in the course of disease, trusting that their veterinarian will partner with them to find the best treatment option for them and their pet. ${ }^{40}$ Earlier intervention may prevent costly emergency visits and possibly lead to better outcomes. In instances when relinquishment or euthanasia are seemingly the only options because of an owner's financial limitations, offering an SoC may provide additional options.

\section{Conclusions}

Enhancing the veterinarian-client relationship is critical for SoC to successfully expand access to veterinary care. As veterinarians, we must be able to facilitate open and compassionate conversations with families about their goals when caring for their pet and be able to discuss without judgment different care options that align with those goals. By giving context to owners, we can empower them to choose the option that is best for them and their pet without feeling any embarrassment, shame, or distress. ${ }^{41}$ To effectively provide an SoC, veterinarians need to focus on efforts that foster effective communication and build strong trusting relationships with clients.

In this context, veterinarians and the veterinary profession as a whole may benefit from shifting the paradigm by which we measure professional accomplishment. A veterinarian who only considers the outcome to be successful when a patient is cured, a chronic condition is managed, or life is extended beyond expectations may feel dejected when these results are not possible. Broadening the definition of a successful outcome to include support of the human-animal bond through effective communication, partnering with families to develop treatment plans that align with their goals for their pets, and providing care that corresponds with owners expectations can enhance professional fulfillment. ${ }^{40,41}$ This philosophy of practice may also help alleviate some of the burnout and moral distress that many veterinarians experience. ${ }^{42,43}$

Practicing an SoC without judgement and with confidence in the options offered increases access to veterinary care and invites clients to make the best decisions for their pets and families. Fostering a strong partnership with our clients improves the client experience, enhances the impact of care, improves our own professional experience as veterinarians, and, most importantly, improves the health and welfare of our patients.

Given that barriers to care exist, we call on veterinarians to move toward offering an SoC that, by embracing a range of care options, can improve access to veterinary care. All options should be presented in terms of their supporting evidence, costs, and other considerations within the context of relationshipcentered care and using best practices for veterinaryclient communication. Not only can an SoC approach address the best interests of patients and the needs and desires of clients, it may also support veterinarians in dealing with some of the increasing stresses of practice. Accomplishing these goals will require efforts from individual practitioners and the profession in general and will, perhaps, require a reassessment of some aspects of veterinary education.

\section{Acknowledgments}

No third-party funding or support was received in connection with the writing or publication of the manuscript. The authors declare that there were no conflicts of interest.

\section{References}

1. AVMA Veterinary Economics Division. Dog ownership and veterinary visits by income bracket. $\mathrm{J} \mathrm{Am} \mathrm{Vet} \mathrm{Med} \mathrm{Assoc}$ 2020;256:282

2. Access to Veterinary Care Coalition. Access to veterinary care: barriers, current practices, and public policy. Available at: trace. tennessee.edu/cgi/viewcontent.cgi? article $=1016 \&$ context $=$ utk_ smalpubs. Accessed May 19, 2020

3. Humane Society of the United States. Keeping pets for life. Available at: www.humanesociety.org/issues/keeping-petslife. Accessed Oct 2, 2020.

4. Stull JW, Shelby JA, Bonnett BN, et al. Barriers and next steps to providing a spectrum of effective health care to companion animals. I Am Vet Med Assoc 2018;253:1386-1389.

5. Fingland RB, Stone LR, Read EK, et al. Preparing veterinary students for excellence in general practice: building confidence and competence by focusing on spectrum of care. $J$ Am Vet Med Assoc 2021; in press.

6. Sidana A, Hernandez DJ, Feng Z, et al. Treatment decisionmaking for localized prostate cancer: what younger men choose and why. Physiol Behav 2016;176:139-148.

7. Pantaleon L. Why measuring outcomes is important in health care. J Vet Intern Med 2019;33:356-362.

8. Toews L. The information infrastructure that supports evidence-based veterinary medicine: a comparison with human medicine. J Vet Med Educ 2011;38:123-134.

9. Sullivant AM, Mackin AJ, Morse D. Strategies to improve case outcome when referral is not affordable. J Vet Med Educ 2020;47:356-364

10. Vandeweerd JM, Vandeweerd S, Gustin C, et al. Understanding veterinary practitioners' decision-making process: implications for veterinary medical education. J Vet Med Educ 2012;39:142-151.

11. McCobb E, Rozanski EA, Malcolm EA, et al. A novel model for teaching primary care in a community practice setting: Tufts at Tech Community Veterinary Clinic. J Vet Med Educ 2018;45:99-107.

12. Wucherer KL, Conzemius MG, Evans R, et al. Short-term and long-term outcomes for overweight dogs with cranial cruciate ligament rupture treated surgically or nonsurgically. $J$ Am Vet Med Assoc 2013;242:1364-1372.

13. Venn EC, Preisner K, Boscan PL, et al. Evaluation of an outpatient protocol in the treatment of canine parvoviral enteritis. J Vet Emerg Crit Care (San Antonio) 2017;27:52-65.

14. Perley K, Burns CC, Maguire C, et al. Retrospective evaluation of outpatient canine parvovirus treatment in a shelterbased low-cost urban clinic. J Vet Emerg Crit Care (San Antonio) 2020;30:202-208.

15. Slater MR, Pailler S, Gayle JM, et al. Welfare of cats 5 to 29 months after perineal urethrostomy. J Feline Med Surg 2020;22:582-588

16. Pailler S, Slater MS, Lesnikowski SM, et al. Outcomes of pyometra in 405 female dogs treated surgically in a non-specialized setting (2017-2019). J Am Vet Med Assoc 2021;in press.

17. Pailler S, Slater M, Lesnikowski S, et al. Outcomes of pyometra in 126 female cats treated surgically in a non-specialized setting (2017-2019). J Am Vet Med Assoc 2021; in press. 
18. Detweiler M. Why the "dental" divides our profession. dvm360 2019;Sep 21. Available at: www.dvm360.com/ view/why-dental-divides-our-profession. Accessed May 20, 2021.

19. WellnessGulf. 10 ways to handle peer pressure in the workplace. Available at: wellnessgulf.com/workplace/10-wayshandle-peer-pressure-workplace/. Accessed Oct 2, 2020.

20. Jack W. Dyess v. Hugh L. Caraway, 190 So2d (666 La App, 1966).

21. Block G. A new look at standard of care. J Am Vet Med Assoc 2018;252:1343-1344.

22. AVMA. Cyberbullying and how to handle it. Available at: avma.org/resources/practice-management/reputation/ cyberbullying-and-how-handle-it. Accessed Oct 2, 2020.

23. Cornell KK, Coe JB, Shaw DH, et al. Investigation of the effects of a practice-level communication training program on veterinary health-care team members' communication confidence, client satisfaction, and practice financial metrics. J Am Vet Med Assoc 2019;255:1377-1388.

24. Lue TW, Pantenburg DP, Crawford PM. Impact of the owner-pet and client-veterinarian bond on the care that pets receive. J Am Vet Med Assoc 2008;232:531-540.

25. Kanji N, Coe JB, Adams CL, et al. Effect of veterinarian-clientpatient interactions on client adherence to dentistry and surgery recommendations in companion-animal practice. $J \mathrm{Am}$ Vet Med Assoc 2012;240:427-436.

26. McDermott MP, Cobb MA, Tischler VA, et al. Evaluating veterinary practitioner perceptions of communication skills and training. Vet Rec 2017;180:305.

27. Mossop L, Gray C, Blaxter A, et al. Communication skills training: what the vet schools are doing. Vet Rec 2015;176:114117.

28. McMurray J, Boysen S. Skills for communicating empathy to companion animal clients. Companion Anim 2017;22:396401.

29. Dysart LMA, Coe JB, Adams CL. Analysis of solicitation of client concerns in companion animal practice. J Am Vet Med Assoc 2011;238:1609-1615.

30. Shaw JR. Four core communication skills of highly effective practitioners. Vet Clin North Am Small Anim Pract 2006;36:385-396.

31. Garrett LD. How to break bad news to clients. Vet Med 2013;308:524-526.
32. Volk JO, Felsted KE, Thomas JG, et al. Executive summary of the Bayer veterinary care usage study. J Am Vet Med Assoc 2011;239:1311-1316.

33. Goldberg K. Issues in serious veterinary illness and endof-life care. In: Kogan L, Blazina C, eds. Clinician's guide to treating companion animal issues. London: Academic Press, 2019.

34. Show A, Englar RE. Evaluating dog- and cat-owner preferences for Calgary-Cambridge communication skills: results of a questionnaire. J Vet Med Educ 2018;45:534-543.

35. Shaw JR, Adams CL, Bonnett BN, et al. Veterinarian-clientpatient communication during wellness appointments versus appointments related to a health problem in companion animal practice. J Am Vet Med Assoc 2008;233:1576-1586.

36. Carney DR, Banaji MR. First is best. PLoS One 2012;7:e35088.

37. Larson RL, White BJ. Importance of the role of the scientific literature in clinical decision making. J Am Vet Med Assoc 2015;247:58-64.

38. Clarke RL. Price transparency: building community trust. Front Health Serv Manage 2007;23:3-12.

39. Coe JB, Adams CL, Bonnett BN. Prevalence and nature of cost discussions during clinical appointments in companion animal practice. J Am Vet Med Assoc 2009;234:1418-1424.

40. Hughes K, Rhind SM, Mossop L, et al. "Care about my animal, know your stuff and take me seriously": United Kingdom and Australian clients' views on the capabilities most important in their veterinarians. Vet $\operatorname{Rec} 2018 ; 183: 534$.

41. Ward E. Pet care shaming: are we discouraging owners from seeking veterinary care? Vet Times (Peterb) 2017; Oct 16. Available at: www.vettimes.co.uk/article/pet-care-shamingare-we-discouraging-owners-from-seeking-veterinary-care/. Accessed Oct 2, 2020.

42. Kipperman BS, Kass PH, Rishniw M. Factors that influence small animal veterinarians' opinions and actions regarding cost of care and effects of economic limitations on patient care and outcome and professional career satisfaction and burnout. J Am Vet Med Assoc 2017;250:785-794.

43. Volk JO, Schimmack U, Strand EB, et al. Executive summary of the Merck Animal Health Veterinary Wellbeing Study. J Am Vet Med Assoc 2018;252:1231-1238.

For all Viewpoint articles, opinions expressed are those of the authors and do not necessarily reflect the official policy of the AVMA 